\title{
Effect of Cilia Orientation in Metachronal Transport of Microparticles
}

\author{
Christoph Brücker ${ }^{1}$, Uwe Schnakenberg ${ }^{2}$, Alexander Rockenbach ${ }^{2}$, Vladimir Mikulich ${ }^{3}$ \\ ${ }^{1}$ Department of Mechanical Engineering and Aeronautics, City University, London, UK \\ ${ }^{2}$ Institute of Materials in Electrical Engineering 1, RWTH Aachen University, Aachen, Germany \\ ${ }^{3}$ Institute of Mechanics and Fluid Dynamics, TU Bergakademie, Freiberg, Germany \\ Email: christoph.bruecker@city.ac.uk
}

How to cite this paper: Brücker, C., Schnakenberg, U., Rockenbach, A. and Mikulich, V. (2017) Effect of Cilia Orientation in Metachronal Transport of Microparticles. World Journal of Mechanics, 7, 1-10.

http://dx.doi.org/10.4236/wjm.2017.71001

Received: December 21, 2016

Accepted: January 10, 2017

Published: January 13, 2017

Copyright (C) 2017 by authors and Scientific Research Publishing Inc. This work is licensed under the Creative Commons Attribution International License (CC BY 4.0).

http://creativecommons.org/licenses/by/4.0/

\section{Open Access}

\begin{abstract}
A biomimetic approach is used to generate a directed transversal transportation of micron-sized particles in liquids based on the principle of cilia-type arrays in coordinated motion. Rows of flaps mimicking planar cilia are positioned off-centre along an array of cavities covered with membranes that support the flaps. These membranes are deflected from a concave to a convex shape and vice versa by pneumatic actuation applying positive and negative pressures (relative to the ambient) inside the cavities. As a result, the flap on top of the membrane tilts to the left or right within such a pressure cycle, performing a beat stroke. Since each cavity can be addressed in the device individually and in rapid succession, waves of coordinated flap motion can be run along the wall. Such metachronal waves are generated and transport of particles along the cilia surface is achieved in both symplectic and antiplectic direction. It is shown that the initial tilt of the flaps relative to the wall-normal determines the direction of transport.
\end{abstract}

\section{Keywords}

Cilia Transport, Metachronal Wave, Fluidic Transport, Micro-Particles

\section{Introduction}

Thanks to the advances in micro- and nanofabrication technologies during the last decades, several micro-manipulation techniques offer the possibility to transport and rotate individual micro-particles or micro-parts. Sorting, trapping, separation, aligning, concentration, patterning, focusing, merging, delivery, and (self-)assembly of micro-objects are of special interest in basic research, development and industrial relevant applications. Different manipulation principles, such as trapping by optical tweezes [1], gripping techniques [2] [3] [4] [5], ul- 
trasonic [6] [7] or magnetic actuation [8] as well as the use of capillary and electrostatic forces for self-assembly of parts [9] are reviewed in detail. The manipulation of particles in microfluidic devices using electrical fields is carried out by electrophoresis or dielectrophoresis actuation principle [10] [11].

When looking to nature, ciliated surfaces are found for transport purposes, such as in the respiratory tract [12] or the fallopian tube [13] or are used for selfpropelling of micro-swimmers [14]. Ciliated walls actuated by applying metachronal waves can be perfectly used for particle transport. The interaction among the particles, the liquid layer and the cilia is coupled by friction at the contacting surfaces, viscous drag and inertia, depending on the local Reynolds number of the cilia beat and the flow around the particles. This raises the question of the effectiveness of metachronal coordination not only for transport of any liquid surrounding the cilia but also for transport of particles itself submerged within the liquid [15] or in contact with the cilia tips. Momentum transfer is possible by direct contact between the particles and the cilia. Different artificial cilia models are already developed to investigate and characterize the transport behavior near the ciliated wall [16]-[26].

The method presented herein uses micro-structured surfaces with arrays of artificial cilia that are individually micro-pneumatically activated [26]. We used spherical micro-particles with a diameter larger than the spacing between the artificial cilia and studied their transport behaviour when metachronal waves running along the ciliated wall are applied. Two different orientations of the cilia in rest conditions were studied to understand the importance of cilia orientation on the direction of transport: the first is with the cilia axes perpendicular to the wall and the second is with a pre-defined tilt.

\section{Methods}

\subsection{Design Concept}

The principle described is to generate coherent transport of spherical particles along a wall covered with artificial cilia, called flaps. The design concept is based upon a model built for liquid flow studies along flexible walls, as illustrated in Figure 1 and Figure 2.

It consists of a pneumatic drive system to generate the movement of flaps in arrays on top of a transportation device (henceforth referred to as port) which is integrated into an open flow chamber at the bottom wall, see Figure 2(a). The port has a total length of $80 \mathrm{~mm}$ and is milled from aluminium. On the surface of the port an array of 20 cavities (spacing $\Delta$ s between each cavity is $1 \mathrm{~mm}$ ) made from PDMS (poly(dimethylsiloxane)) is located, each one covered with a flexible membrane. Each membrane has a flap on top of it (height $500 \mu \mathrm{m}$, thickness $50 \mu \mathrm{m}$, extension of the flap span in direction normal to the paperplane $30 \mathrm{~mm}$ ) which protrudes into the liquid layer at a defined angle relative to the membrane. The flap orientation at ambient pressure (planar membrane) is either perpendicular as in modification 1 or tilted at $45^{\circ}$ as in modification 2 , see Figure 1. A picture of the membrane is shown in Figure 2(b). The port houses 
Modification 1

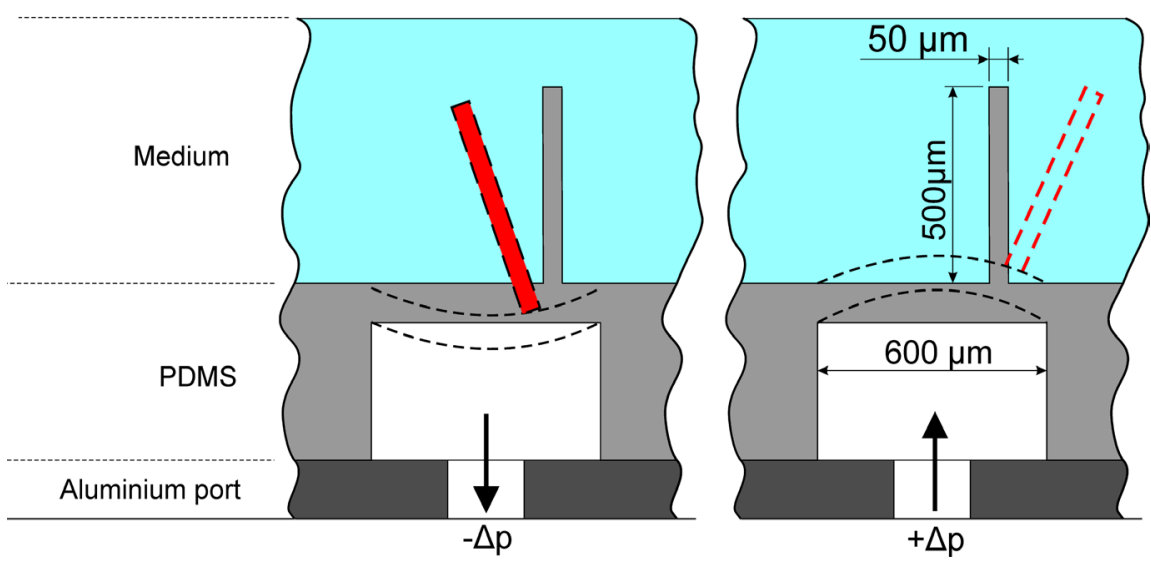

(a)

Modification 2

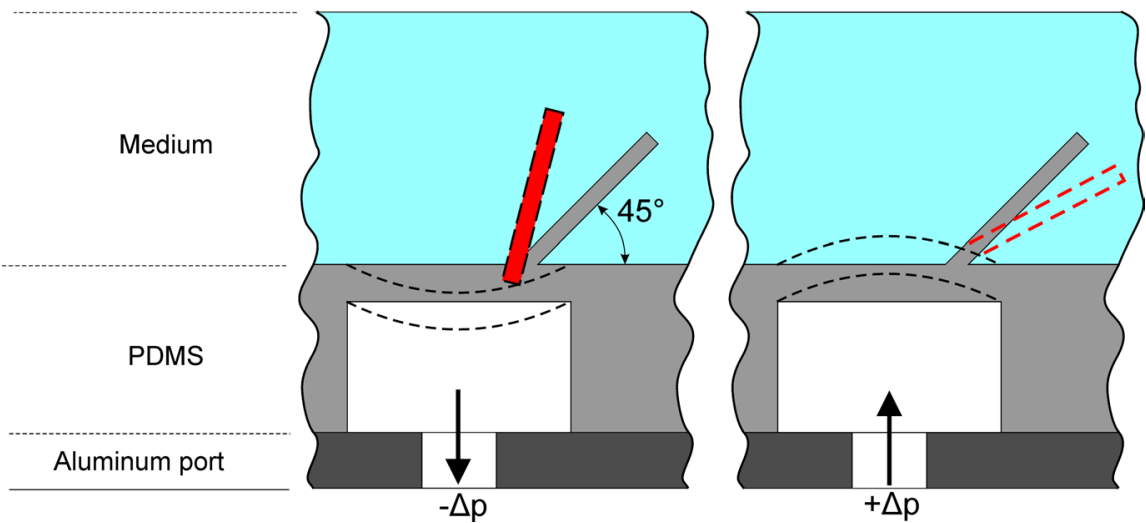

(b)

Figure 1. Schematic of the pneumatic flap actuation mechanism in two modifications of the flaps. The solid contours represent the state at ambient pressure $\Delta \mathrm{p}=0$ where the membrane is flat. The dashed lines display the state of the membrane and the flap at the different pressure conditions at $\pm \Delta \mathrm{p} \neq 0$ with flap colored in solid red for the ground state at $-\Delta$ p. (a) Flaps have at the ground state a negative tilt (against beat direction) of $22.5^{\circ}$ against the wall-normal. (b) Flaps have at the ground state a positive tilt (within beat direction) of $+22.5^{\circ}$ against the wall-normal. The flap angle for $\Delta \mathrm{p}=0$ can be adjusted in the manufacturing process of the membrane.

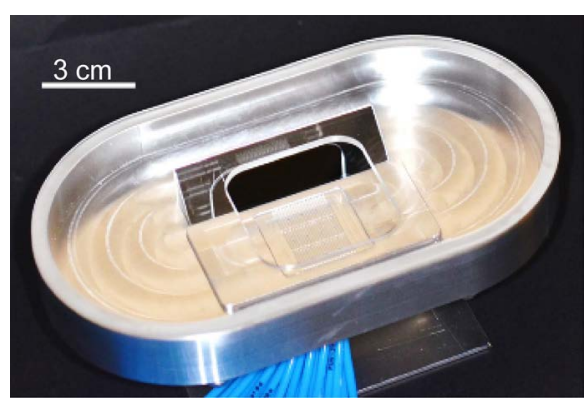

(a)

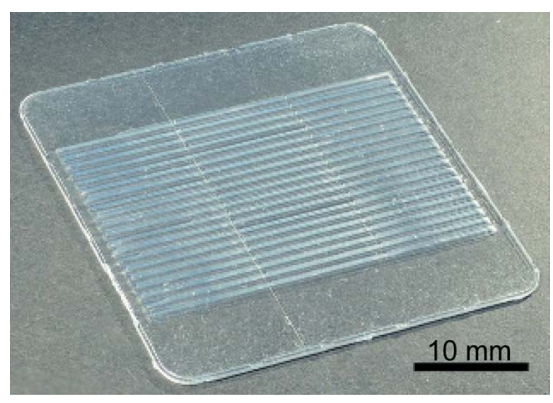

(b)

Figure 2. (a) Closed-loop flow chamber. The silicon device glued to the connector is integrated into the chamber. The channels under the membrane are connected to the blue pressure tubes. (b) Photograph of the upper membrane with the 20 transparent flaps arranged in rows with a spacing of $\Delta \mathrm{s}=1 \mathrm{~mm}$. 
pneumatic connectors on its bottom for the attachment of pneumatic tubes. These tubes deliver either positive or negative pressure (relative to the ambient) to each cavity which is switched by magnetic valves. The ground state in the experiments is when negative pressure is applied to the cavities, and then all membranes are in concave state. A change in pressure from negative to positive in one cavity results in change of the curvature of the membrane from the concave state to the convex state. This causes the local flap to tilt to the right, performing a beat stroke. When the valve switches back to the ground state the flap relaxes back to its original position. The control of all 20 flaps to beat in metachronal wave-like pattern is explained further below.

\subsection{Experimental Set-Up}

The port with the ciliated membrane is integrated at the bottom of an open channel which is filled with water (see Figure 2). The open channel is $10 \mathrm{~mm}$ deep and is made of aluminum. It consists of two straight parts connected by semi-circular arches. Transparent glass windows are implemented into the sides of the channel walls to allow optical access with a high-speed camera (HS-C2 in Figure 3). An optional second camera can look via a $45^{\circ}$ prism from top onto the ciliated wall (HS-C1 in Figure 3). The cameras are synchronized with each other while recording. Illumination is done with a LED-lighting system.

The driving unit to generate the metachronal waves is depicted in Figure 3. To control the movement of every flap individually via the pneumatic system, a rail of 20 valves is used with the abovementioned pneumatic tubes connected to the port cavities. On one side the valves are connected to a pressurised air supply (positive pressure supply) and on the other side the valves are connected to a vacuum pump (negative pressure supply). The positive and negative relative

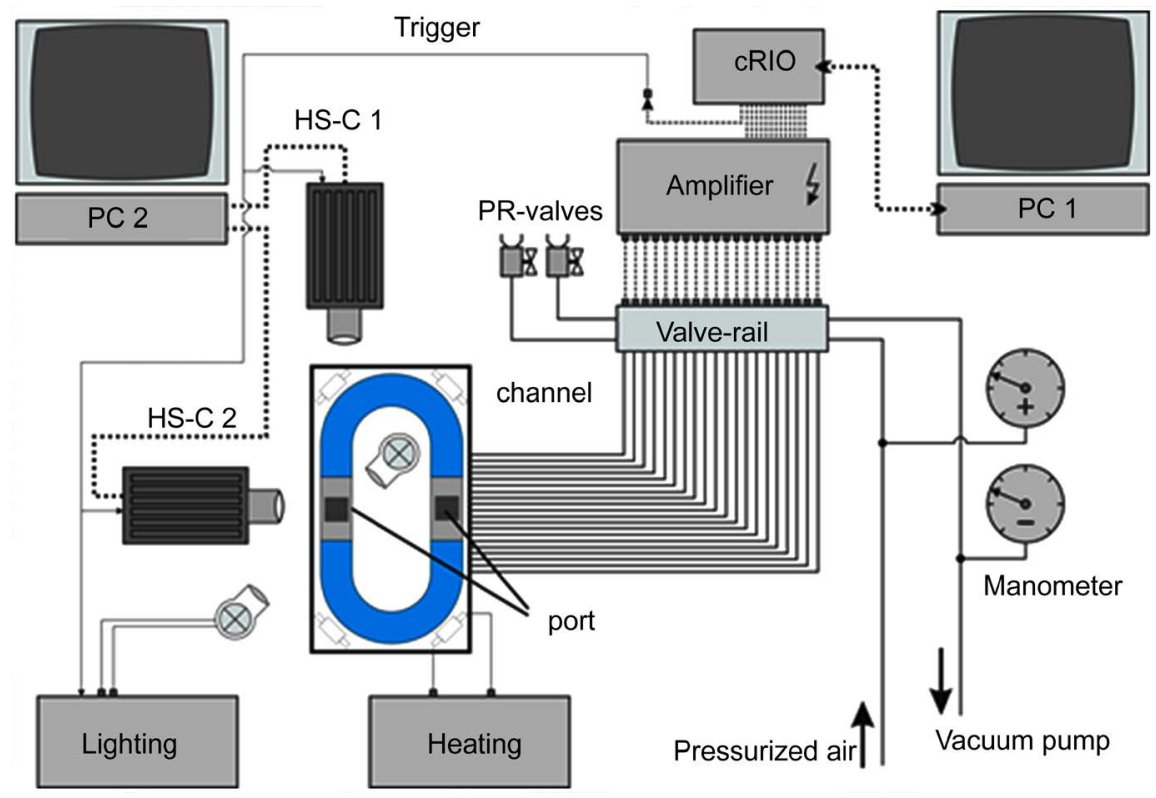

Figure 3. Schematic block diagram of the control and data acquisition setup, with a detailed view of the pneumatic design concept. 
pressures are monitored with two separate manometers. A Personal Computer with an analogue input/output controller (cRIO National Instruments) is used to trigger the valves. The controller has its own processor and a reconfigurable Field Programmable Gate Array (FPGA). The latter ensures that the execution of the control algorithms occurs in real-time. An additional amplifier is needed to amplify and transfer the signal to each valve.

The design concept has the following parameters to generate a particular form of metachronal waves: the wavelength which is equal to the pulse repetition length $\mathrm{L}$ along the flap array, the pulse propagation speed $U$ and the number of neighbouring cavities which are active in a single pulse. This is visualised in Figure 4. Once the wave reaches the end, it is reintroduced at the beginning of the array.The time the valves need to switch from negative pressure to positive one and vice versa is approximately $4 \mathrm{~ms}$. In comparison, for the flap it takes about $40 \mathrm{~ms}$ to move from one side to the other in such a pressure pulse. This is the consequence of the damping in the pressure lines which cause a delay of the pressure built up or decay under the membrane until it reaches the maximum or minimum value [26]. In the following, this period is referred as the characteristic beating time of a flap $t_{B}$. Another time-scale is attributed to the wave-cycle time $T$ (inverse of the wave repetition frequency) which is the time between successive pulses at the same cavity and reads $T=L / U$.

\subsection{Experimental Procedure}

For all tests, the channel is filled with distilled water. It is held at a temperature of $24{ }^{\circ} \mathrm{C}$. To be certain of an equal temperature distribution the channel, water and ports were monitored with an infrared camera. The water layer thickness

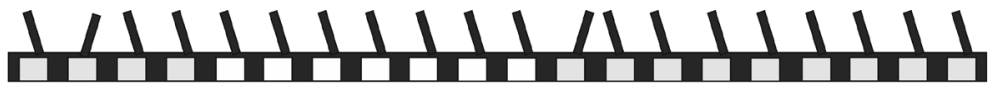

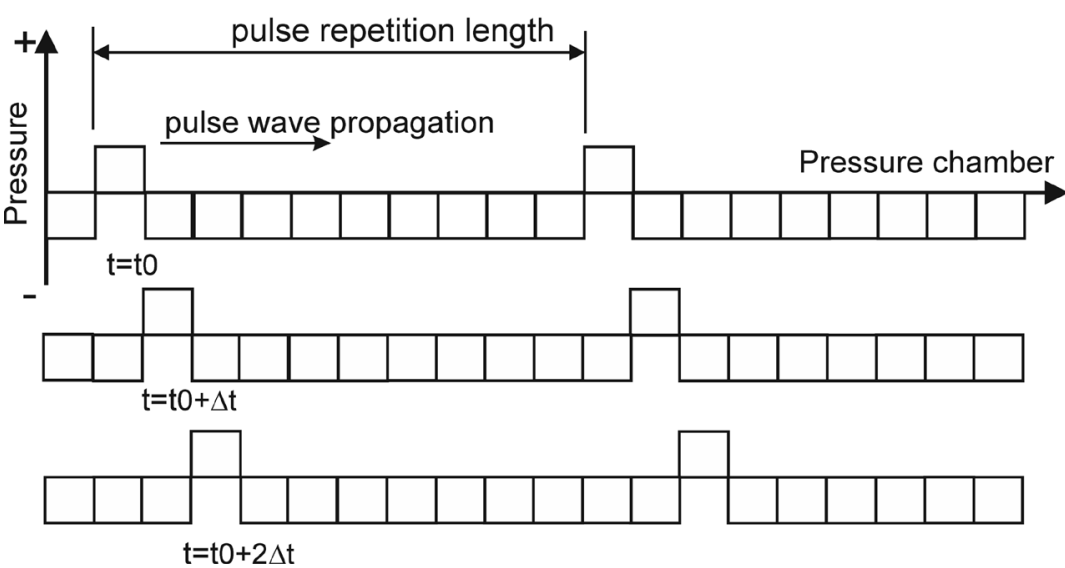

Figure 4. Principle of the wave generation with the controlled switching of the pressures in the cavities below the array of flaps. The propagation direction is from left to right. In the picture, the number of active cavities in a single pulse is the minimum of one. There is also the possibility to activate two or three neighbouring cavities to participate in a pulse. The flaps corresponding to the pulse are called the "active" flaps. The pulse pattern progresses through the array in time steps of $\Delta t$. 
was kept at $3 \mathrm{~mm}$ throughout all experimental procedures. Although two ports with different modifications respectively are mounted in the channel, only one is used herein. Spherical polyamide particles with a density of $1.14 \mathrm{~g} / \mathrm{cm}^{3}$ are used in the experimental procedure. The diameter was chosen to $2 \mathrm{~mm}$ to ensure that the particles do not fall into the gap between the flaps and remain in contact with their tips. The available spherical polyamide particles are colored in white first to achieve a good visibility in the recordings. To detect any rotational movement, their surfaces were marked with irregular black dots in a second step. For each test the particles were carefully placed between the first and second flap on one side of the array. The particle motion was recorded with the CCD camera simultaneous from the side and from the top to determine their paths along the ciliated array. A particle tracking software was used to process the time-series of images from the recordings and to determine the particle tracks.

The test series were conducted with an applied pressure of 0.4 bar in positive and negative direction relative to the ambient pressure. Initial parametric studies have shown that at least \pm 0.2 bar pressure has to be applied to the flaps to ensure the transport of the particles. At lower pressures, the momentum transferred to the particles is not sufficient to move the particles from one flap to the next.

\section{Results}

The focus of our studies is the transport process for the two different flap orientations. The difference between both settings is explained in Figure 1. The particle transport in general depends on a large set of parameters such as the Reynolds number of the flow around the particles, the density ratio between particle and fluid, the pulse pattern and wave speed as well as the shape and size of the particles. A study of all parameters is beyond the scope of the paper; rather we selected characteristic results to demonstrate the impact of cilia orientation on the transport direction under otherwise constant conditions.

\subsection{Symplectic Transport}

Figure 5 shows the symplectic transport of the sphere with the flap modification 1 (flaps have at ground state a negative tilt to the left against beat direction) when the pressure pulse propagation is applied from left to right. The beat cycle is when the flap tip rotates from left to right in clock-wise direction (red position in Figure 5) and relaxes back passing the blue state in Figure 5 until it ends again at the original state (black position in Figure 5). In the symplectic transport, the momentum transfer occurs mainly via a contact between the sphere and the side wall of the flap. The major phase in momentum transfer is the forward-directed stroke of the flap. The larger contact area at the side wall of the flap compared to the tip area allows a more efficient horizontal transportation, resulting in a high particle velocity of $12 \mathrm{~mm} / \mathrm{s}$. Furthermore, the sphere gets a positive spin. Overall, the sphere leads the front crest of the travelling wave and is a fast as the wave speed is. Note that reversing the propagation direction from right to left does not lead to a transport at all. 


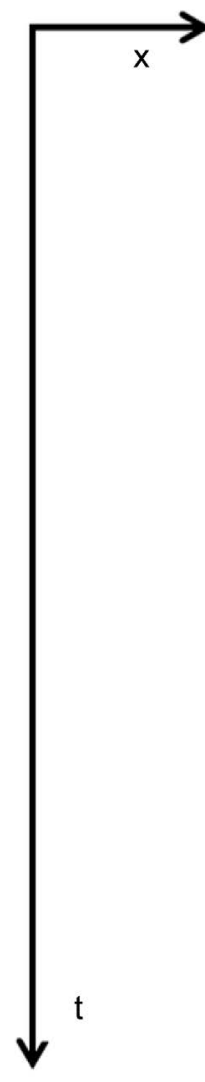

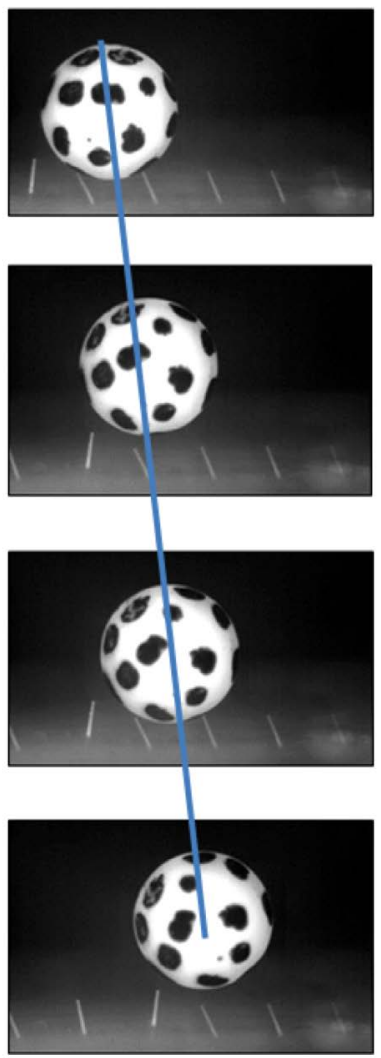

(a)

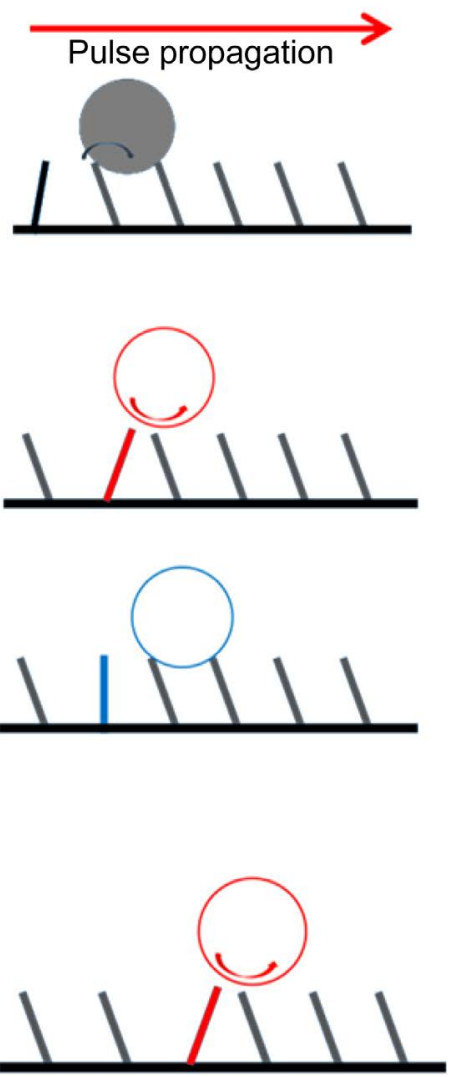

(b)

Figure 5. Symplectic transport of a sphere along the ciliated wall over time t. Pulse propagation is from left to right in positive $\mathrm{x}$-direction at a speed of $12 \Delta \mathrm{s} / \mathrm{sec}(12 \mathrm{~mm} / \mathrm{sec})$. Particle transport is also in positive x-direction and mean particle speed is $U_{P}=U$. Spin of the particle is counter-clockwise. Number of active flaps in a pulse is one.

\subsection{Antiplectic Transport}

In comparison, Figure 6 shows an example where an antiplectic transport is observed for flap modification 2 (flaps have at ground state a positive tilt within beat direction of $+22.5^{\circ}$ ). Note that for these experiments the pressure pulse propagation is from the right to the left and was set to the same velocity as in the experiments with flap modification 1 . In this case, the number of active flaps involved in the momentum transfer was increased to two, which gave the best results for transport. The beat cycle leads to the simultaneous tilt of two neighboring flaps which are in contact with the sphere. The tilt causes the sphere to move to the right down into the gap on the right-hand side of the flap activated in the previous beat. This is because the latter is relaxing to its original position. Within this motion phase of the sphere the right-hand sided active flap is starting to relax back which supports the further motion in combination with the additional impact of a negative spin. This causes the sphere to roll over to the tip of the flap to the next gap on the right-hand side. In this way, the sphere is shifted a distance of $\Delta$ s per wave cycle in direction counter to the wave propagation. Again, a reversal of the propagation direction from left to right does not show a transport at all for this configuration. 


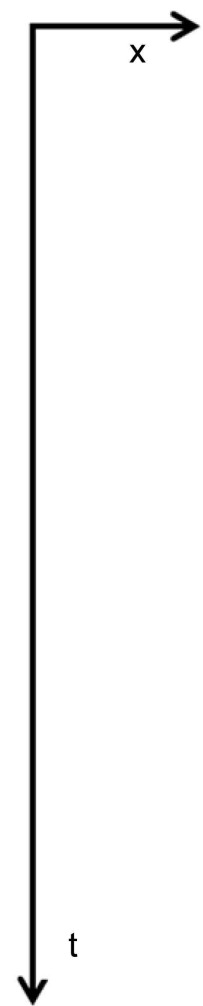

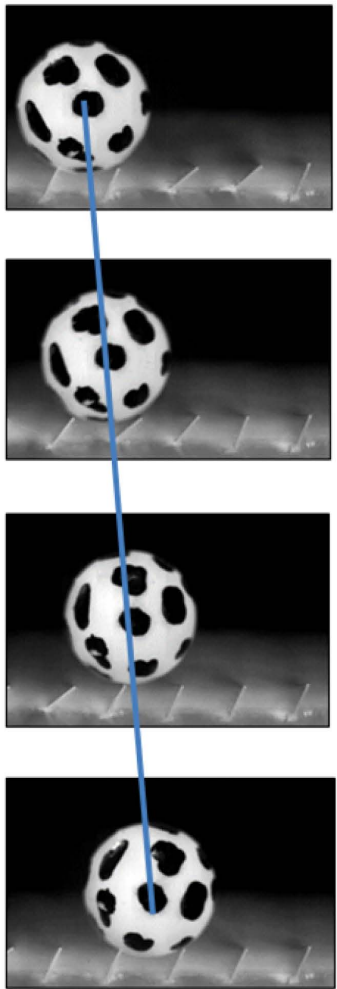

(a)
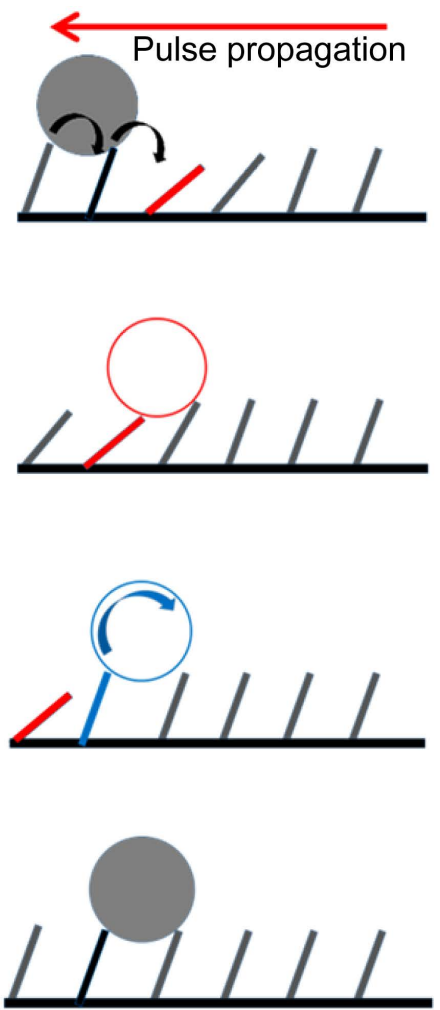

(b)

Figure 6. Antiplectic transport of a sphere along the ciliated wall over time t. Pulse propagation is in negative $\mathrm{x}$-direction from right to left at a speed of $12 \Delta \mathrm{s} / \mathrm{sec}(12 \mathrm{~mm} / \mathrm{sec})$. Particle transport is in positive x-direction at a speed of $U_{P}=\Delta s / T$ which is one flap per wave cycle. Spin of the particle is clockwise. Number of active flaps in a pulse is two.

A successful transport depends primarily on the momentum transfer of the flaps onto the particle and the wave pattern. It is crucial for maximum transport velocity how the particles are positioned before they receive the impulse and in which manner the particles travel after receiving the impulse. A logical pattern is a shift of one flap per beat cycle so that the next flap takes over the sphere and pushes it further forward. In this case the transport is symplecticin the same direction as the wave propagates and reaches maximum speed. However, this transport requires an initial negative pre-tilt of the flaps against the beat direction as shown in Figure 1(a), otherwise the transport does not happen. If the direction of the pre-tilt is with direction of the beat (Figure 1(b)), particle transport is only observed if the wave propagation direction is reversed. This means an antiplectic transport in the metachronal wave.

\section{Conclusion}

The transportation of particles along ciliated walls is of highly complex nature. In order to simplify the parameter space, an artificially ciliated wall is designed which allows us to study the transport of selected particles under defined beating conditions. As reference, we selected spherical particles of millimeter-size in an aqueous carrier liquid. The particle diameter is two-times larger than the spacing 
between the flaps to ensure contact with the flap tips. In addition, their density of $1.14 \mathrm{~g} / \mathrm{cm}^{3}$ is slightly above that of water to keep the particles in contact with the ciliated wall which is arranged in the horizontal plane. The results show that the pre-tilt of the flaps against the wall-normal is an important parameter which decides about the transport direction. Symplectic transport is observed herein for a pre-tilt of the cilia against the beating direction while antipletic transport is observed for a pre-tilt in direction of the cilia beat. This may explain discrepancies in observations of transport along ciliated walls depending on the arrangement and orientation of the hairy structures.

\section{Acknowledgements}

Funding is given partly by the German Federal Ministry of Education and Research under grant \#16SV5341 and the Deutsche Forschungsgemeinschaft (DFG) in the SPP "Microswimmer". The contribution by Vladimir Mikulich was granted by the DFG in the project BR 1494/20-2. Funding of the position of Professor Christoph Bruecker as the BAE SYSTEMS Sir Richard Olver Chair in Aeronautical Engineering is gratefully acknowledged herein.

\section{References}

[1] Grier, D.G. (2003) A Revolution in Optical Manipulation. Nature, 242, 810-816.

[2] Cecil, J., et al. (2007) Assembly and Manipulation of Micro Devices-A State of the Art Survey. Robotics and Computer-Integrated Manufacturing, 23, 580-588. https://doi.org/10.1016/j.rcim.2006.05.010

[3] Vasquez, D., et al. (2007) A Review of Gripping and Manipulation Techniques for Micro-Assembly Applications. International Journal of Production Research, 43, 819-828.

[4] Ouyang, P.R., et al. (2006) Micro-Motion Devices Technology: The State of Arts Review. International Journal of Advanced Manufacturing Technology, 38, 463478.

[5] Gauthier, M., et al. (2006) Analysis of Forces for Micromanipulation in Dry and Liquid Media. Journal of Micromechatronics, 3, 389-413. https://doi.org/10.1163/156856306777924699

[6] Drinkwater, B.W. (2016) Dynamic-Field Devices for the Ultrasonic Manipulation of Microparticles. Lab on a Chip, 16, 2360-2375. https://doi.org/10.1039/C6LC00502K

[7] Destgeer, G., et al. (2015) Recent Advances in Microfluidic Actuation and MicroObject Manipulation via Surface Acoustic Waves. Lab on a Chip, 15, 2722-2738. https://doi.org/10.1039/C5LC00265F

[8] Gijs, M.A.M. (2004) Magnetic Bead Handling On-Chip: New Opportunities for Analytical Applications. Microfluidics and Nanofluidics, 1, 22-40.

[9] Mastrangeli, et al. (2009) Self-Assembly from Milli- to Nanoscales: Methods and Applications. Journal of Micromechanics and Microengineering, 19, 083001. https://doi.org/10.1088/0960-1317/19/8/083001

[10] Pethig, R. (2010) Review Article-Dielectrophoresis: Status of the Theory, Technology and Applications. Biomicrofluidics, 4, 022811.

[11] Velev, O.D., et al. (2009) Particle-Localized AC and DC Manipulation and electrokinetics. Annu Rep Prog Chem, Sect. C, 105, 213-246. 
[12] Fulford, G.R., et al. (1986) Mucociliary Transport in the Lung. Journal of Theoretical Biology, 121, 381-402. https://doi.org/10.1016/S0022-5193(86)80098-4

[13] Lyons, R.A., et al. (2006) The Reproductive Significance of Human Fallopian Tube Cilia. Human Reproduction Update, 12, 363-372. https://doi.org/10.1093/humupd/dml012

[14] Elgeti, J., et al. (2015) Physics of Microswimmers-Single Particle Motion and Collective Behaviour: A Review. Reports on Progress in Physics, 78, 056601. https://doi.org/10.1088/0034-4885/78/5/056601

[15] Masoud, H., et al. (2011) Harnessing Synthetic Cilia to Regulate Motion of Microparticles. Soft Matter, 7, 8702-8708.

[16] den Toonder, J.M., et al. (2008) Artificial Cilia for Active Micro-Fluidic Mixing. Lab on a Chip, 8, 533-541. https://doi.org/10.1039/b717681c

[17] den Toonder, J.M.J., et al. (2013) Microfluidic Manipulation with Artificial/Bioinspired Cilia. Trends in Biotechnology, 31, 85-91. https://doi.org/10.1016/j.tibtech.2012.11.005

[18] Khaderi, S.N., et al. (2011) Magnetically-Actuated Artificial Cilia for Microfluidic Propulsion. Lab on a Chip, 11, 2002-2010. https://doi.org/10.1039/c0lc00411a

[19] Shields, A.R., et al. (2010) Biomimetic Cilia Arrays Generate Simultaneous Pumping and Mixing Regimes. Proceedings of the National Academy of Sciences of the United States of America, 107, 15670-15675. https://doi.org/10.1073/pnas.1005127107

[20] Fahrni, F., et al. (2009) Micro-Fluidic Actuation Using Magnetic Artificial Cilia. Lab on a Chip, 9, 3413-3421. https://doi.org/10.1039/b908578e

[21] Timonen, J.V.I., et al. (2010) A Facile Template-Free Approach to Magnetodriven, Multifunctional Artificial Cilia. ACS Applied Materials \& Interfaces, 2, 2226-2230. https://doi.org/10.1021/am100244x

[22] van Oosten, C.L., et al. (2009) Printed Artificial Cilia from Liquid-Crystal Network Actuators Modularly Driven by Light. Nature Materials, 8, 677-682.

https://doi.org/10.1038/nmat2487

[23] Zarzar, L.D., et al. (2011) Bio-Inspired Design of Submerged Hydrogel-Actuated Polymer Microstructures Operating in Response to pH. Advanced Materials, 23, 1442-1446. https://doi.org/10.1002/adma.201004231

[24] Sanchez, T., et al. (2011) Cilia-Like Beating of Active Microtubule Bundles. Science, 333, 456-459.

[25] Dayal, P., et al. (2012) Chemically-Mediated Communication in Self-Oscillating, Biomimetic Cilia. Journal of Materials Chemistry, 22, 241. https://doi.org/10.1039/C1JM13787E

[26] Rockenbach, A., et al. (2015) Fluid Transport via Pneumatically Actuated Waves on a Ciliated Wall. Journal of Micromechanics and Microengineering, 25, 125009. https://doi.org/10.1088/0960-1317/25/12/125009 
Submit or recommend next manuscript to SCIRP and we will provide best service for you:

Accepting pre-submission inquiries through Email, Facebook, LinkedIn, Twitter, etc. A wide selection of journals (inclusive of 9 subjects, more than 200 journals)

Providing 24-hour high-quality service

User-friendly online submission system

Fair and swift peer-review system

Efficient typesetting and proofreading procedure

Display of the result of downloads and visits, as well as the number of cited articles Maximum dissemination of your research work

Submit your manuscript at: http://papersubmission.scirp.org/

Or contact wjm@scirp.org 